\title{
IMPACTO DA ULTRA-SONOGRAFIA INTRA-OPERATÓRIA NAS CIRURGIAS PARA RESSECÇÃO DE METÁSTASES HEPÁTICAS
}

\author{
Marcela Pecora COHEN ${ }^{1}$, Marcel Autran Cesar MACHADO ${ }^{2}$ e Paulo HERMAN ${ }^{2}$
}

RESUMO - Racional - Vinte e cinco a 50\% dos pacientes com metástases hepáticas são potenciais candidatos à cirurgia curativa. A ultra-sonografia intra-operatória tem sido usada com grande acurácia para orientar a ressecção das lesões hepáticas. Objetivo - Avaliar em nosso meio a importância desse método nas cirurgias hepáticas e comparar seus achados com os dos métodos de imagem pré-operatórios. Pacientes $\boldsymbol{e}$ Métodos - Estudou-se prospectivamente 35 pacientes com metástases hepáticas e indicação cirúrgica, com os seguintes tumores primários: cólon (24), tumor neuroendócrino (3), carcinoma de células renais e melanoma (2) e outros (4). A idade mediana foi de 56 anos, sendo 20 pacientes do sexo feminino. Os pacientes foram submetidos a pelo menos um exame entre tomografia computadorizada (30), ultra-sonografia (14) e ressonância magnética (8). Resultados - A ultra-sonografia intra-operatória foi útil em 23 (65,6\%) das 35 cirurgias, e mudou o plano cirúrgico em $9(25,7 \%)$ pacientes. Houve correlação estatisticamente significativa entre o número de nódulos identificados por ultrasonografia percutânea do abdome, tomografia computadorizada e ressonância magnética, e a ultra-sonografia intra-operatória, sendo que a média de nódulos por paciente encontrados pela ressonância magnética $(2,6)$ e pela ultra-sonografia intraoperatória $(3,0)$ não mostrou diferença estatisticamente significativa. Cinqüenta e cinco nódulos foram submetidos a análise anatomopatológica, considerada padrão-ouro para o cálculo de sensibilidade da ultra-sonografia intra-operatória, sendo que $52(94,5 \%)$ foram identificados pela ultra-sonografia intra-operatória. Dos nódulos menores que 1,5 cm, a ultra-sonografia detectou $15,0 \%$, a tomografia computadorizada $33,3 \%$, a ressonância magnética $66,6 \%$ e a ultra-sonografia intra-operatória 91,6\%. Conclusões - A ultra-sonografia intra-operatória é útil na avaliação e conduta intra-operatórias durante as ressecções de metástases hepáticas, especialmente em pacientes com nódulos pequenos.

DESCRITORES - Metástase neoplásica, ultra-sonografia. Neoplasias hepáticas, cirurgia.

\section{INTRODUÇÃO}

Dentre os tumores malignos do fígado, as lesões metastáticas são as mais comuns e sua presença está associada a mau prognóstico, que pode ser melhorado através do emprego de terapias agressivas, como a ressecção hepática.

Os pacientes com câncer e lesões secundárias no fígado que podem se beneficiar da ressecção das metástases, são aqueles com carcinoma colorretal (CCR), tumores neuroendócrinos e carcinoma renal ${ }^{(7)}$. Entre esses, os mais estudados são os com metástase de CCR, pela sua maior freqüência, com índices de sobrevida que seriam de 8 a 9 meses sem tratamento cirúrgico, podendo atingir $40 \%$ a $50 \%$ em 5 anos após a ressecção ${ }^{(9,10)}$.

Para metástases hepáticas de tumores neuroendócrinos são descritos índices de sobrevida de 5 anos acima de $60 \%$ após a ressecção e, mesmo quando esta não é curativa, a cirurgia oferece excelente controle da doença ${ }^{(29)}$. Em pacientes com metástases de tumores renais, a cirurgia é considerada a única chance de cura, com índices de sobrevida de 5 anos de cerca de $20 \%{ }^{(1)}$. Dentre os outros tumores metastáticos para o fígado, estão ainda em estudo dentro de protocolos, os benefícios da ressecção para metástases de tumores de mama, testículo, sarcomas, papila, melanoma e tumores ginecológicos, com índices de sobrevida que podem alcançar $20 \%{ }^{(7,8,31)}$.

O diagnóstico das metástases hepáticas baseia-se no rastreamento com exames radiológicos, uma vez que sinais e sintomas clínicos e a elevação das enzimas hepáticas ocorrem tardiamente, quando o paciente já não tem mais chance de cura através do tratamento cirúrgico. Entre os métodos de imagem mais difundidos destacam-se a ultra-sonografia percutânea do abdome (US), a tomografia computadorizada (TC) e a ressonância nuclear magnética do abdome (RNM). 
A US é um método de baixo custo, grande disponibilidade e sem riscos ao paciente, com sensibilidade ao redor de $55 \%{ }^{(13)}$. Atualmente, a TC é o método mais empregado para o rastreamento de metástases hepáticas com sensibilidade de cerca de $72 \%$, variando entre $36 \%$ e $96 \%$, sendo mais alta com o emprego de equipamentos helicoidais ${ }^{(13,36)}$. A RNM apresenta acurácia semelhante à da TC na detecção de lesões focais hepáticas, com sensibilidade em torno de $76 \%$ e especificidade da ordem de $97 \%$, sensivelmente mais alta que a de outros métodos ${ }^{(11,13,32)}$. No entanto, é sabido que os exames de imagem disponíveis não são capazes de demonstrar com precisão lesões menores que $1,5 \mathrm{~cm}$, uma vez que lesões hepáticas pequenas estão abaixo do nível de resolução desses métodos ${ }^{(17)}$.

Para a avaliação e conduta cirúrgica mais adequadas, é de fundamental importância a determinação precisa do número e da localização anatômica dessas lesões. Essas informações, fornecidas pelos métodos de imagem pré-operatórios, devem ser complementadas através da avaliação intra-operatória, que inclui a inspeção e palpação pelo cirurgião e, a ultra-sonografia intra-operatória (USIO).

A USIO hepática apresenta vantagens em relação aos outros métodos de diagnóstico por imagem, pois detecta maior número de lesões (sensibilidade entre $80 \%$ e $98,5 \%$ ), especialmente as pequenas e, em número considerável de casos, muda o plano cirúrgico e/ou o estádio da doença ${ }^{(33)}$. Por esses motivos, tornou-se obrigatória nos centros especializados em cirurgia hepática ${ }^{(3,16)}$. Na literatura internacional publicações que envolvem a USIO são freqüentes; no Brasil porém, pouco se tem estudado sobre o assunto ${ }^{(15,16)}$.

O objetivo principal deste trabalho foi verificar a importância da USIO no tratamento cirúrgico dos pacientes submetidos a laparotomia para ressecção de metástases hepáticas, no Centro de Tratamento e Pesquisa do Hospital do Câncer A. C. Camargo, São Paulo, SP, onde é empregada de forma rotineira.

O objetivo secundário é comparar os achados da USIO aos dos exames de imagem pré-operatórios (US, TC e RNM) e ao estudo anatomopatológico dos segmentos hepáticos ressecados.

\section{MATERIAL E MÉTODOS}

Neste estudo prospectivo, foram estudados 35 pacientes com diagnóstico de metástases hepáticas, com indicação de ressecção e submetidos a cirurgia, no período entre fevereiro de 2001 e julho de 2003.

Entre os 35 pacientes, 15 (42,8\%) eram do sexo masculino e $20(57,2 \%)$ do feminino, com idade entre 33 e 80 anos (mediana de 56 anos). Vinte e quatro $(68,5 \%)$ pacientes tinham como doença de base câncer colorretal, três $(8,5 \%)$ tumor neuroendócrino, dois $(5,7 \%)$ melanoma, carcinoma de células renais e sarcomas, e os demais, adenocarcinomas de papila e mama.

O diagnóstico de lesões hepáticas foi baseado nos exames de imagem trazidos pelos pacientes ou realizados no Departamento de Diagnóstico por Imagem do Hospital A. C. Camargo e, dentre os exames radiológicos, considerou-se um ou mais dos seguintes: US em 14 pacientes (40\%), TC em 30 (85,7\%) e RNM em 8 $(22,8 \%)$. Foram considerados para o estudo os exames de boa qualidade realizados no máximo 10 semanas antes da cirurgia.
Os exames de imagem pré-operatórios não foram padronizados, uma vez que parte deles foi trazida de outros serviços.

A análise de US, TC e RNM pré-operatórios foi feita através da identificação de nódulos suspeitos de malignidade ou não e suas características quanto ao número total e dimensões. Após a avaliação conjunta dos exames pelo radiologista e pelo cirurgião, foi traçado um plano cirúrgico para cada paciente, antes da avaliação intra-operatória. Durante a cirurgia, após a inspeção, palpação e USIO, o plano cirúrgico inicial foi reavaliado e, se necessário, modificado.

A USIO foi realizada no ato operatório por radiologista com experiência em ultra-sonografia $(80 \%$ pelo mesmo radiologista), com conhecimento prévio dos exames de imagem pré-operatórios e dos achados de inspeção e palpação cirúrgicas. Foi utilizado equipamento Toshiba Tosbee ${ }^{\circledR}$ (Japão) com transdutor transoperatório em "T" de $7 \mathrm{mHz}$ encapado com plástico esterilizado.

As cirurgias realizadas foram: $10(28,5 \%)$ segmentectomias regradas, $7(20 \%)$ hepatectomias direitas, $3(8,6 \%)$ hepatectomias esquerdas, lobectomias esquerdas, nodulectomias e a combinação de hepatectomia e ablação tumoral, 2 (5,7\%) ablações tumorais por radiofreqüência e em $4(11,4 \%)$ pacientes a hepatectomia foi suspensa.

As lesões foram medidas e a relação destas com as estruturas vasculares hepáticas foi observada, utilizando-se técnicas de palpação conjuntamente com a USIO, citadas recentemente por TORZILLI e MAKUUCHI (35) (Figura 1). A USIO foi também utilizada como guia para biopsia e/ou procedimentos de ablação tumoral como radiofreqüência e/ou alcoolização das lesões.

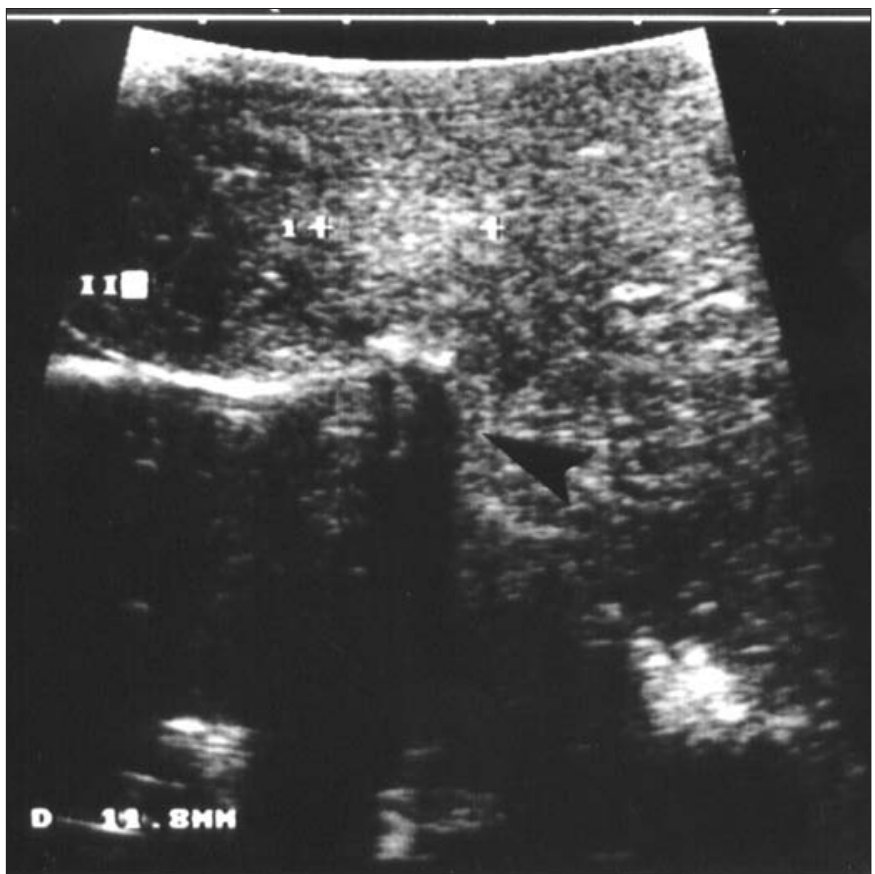

FIGURA 1 - Técnica de palpação conjunta. A USIO detectou nódulo hiperecogênico e com limites imprecisos, que foi confirmado pela palpação. A ponta de seta indica o dedo do cirurgião junto ao nódulo descrito. 
A mesma análise em relação ao número e dimensões das lesões hepáticas realizada para os exames pré-operatórios, foi empregada para a inspeção e palpação bi-manual pelo cirurgião e para a USIO.

Os segmentos hepáticos ressecados foram submetidos a exame histológico e o número e dimensões das lesões hepáticas encontradas também foram analisados, tendo sido este o método considerado como padrão-ouro para o cálculo de sensibilidade da USIO.

Dentre as variáveis do estudo, analisaram-se as características cirúrgicas (mudança do plano cirúrgico, causa desta mudança e o papel da USIO no procedimento cirúrgico) e dos métodos de exames de imagem pré-operatórios e seus achados (número de nódulos por exame, medidas e diagnóstico das lesões).

Foi realizada a análise descritiva de freqüências na avaliação das características cirúrgicas e dos métodos de imagem. Para comparação entre os achados dos exames de imagem pré-operatórios e da USIO foi aplicado o teste $t$ de Student de amostras pareadas e calculado o coeficiente de correlação intra-classe. Para análise nódulo a nódulo, baseada nos achados anatomopatológicos, utilizou-se a estatística descritiva de freqüências.

O trabalho foi submetido e aprovado pelo Comitê de Ética em Pesquisa da instituição e os pacientes assinaram termo de consentimento pós-informado aprovado pela referida Comissão.

\section{RESULTADOS}

A comparação do plano cirúrgico proposto antes da avaliação intra-operatória com o plano estabelecido após tal avaliação mostrou mudança deste em 15 (42,8\%) dos 35 pacientes, sendo que em $9(25,7 \%)$ a mudança se deu devido às informações fornecidas somente pela USIO (Tabela 1).

TABELA 1 - Distribuição dos pacientes em relação à mudança do plano cirúrgico pré-operatório após a avaliação intra-operatória através da palpação, inspeção e ultra-sonografia intraoperatória (USIO)

\begin{tabular}{lcl}
\hline Mudança do plano cirúrgico & $\mathbf{N}^{\circ}$ de pacientes $(\%)$ \\
\hline Não houve & $20(57,1)$ \\
Baseada na USIO & $9 \quad(25,7)$ \\
Baseada na inspeção e palpação & $6(17,2)$ \\
\hline TOTAL & $35 \quad(100)$ \\
\hline
\end{tabular}

Independentemente de ter ou não levado a alguma mudança no plano cirúrgico, em $23(65,7 \%)$ pacientes a USIO foi de alguma forma útil na avaliação intra-operatória: em $16(45,7 \%)$ pacientes forneceu informações relevantes quanto à localização precisa do nódulo e sua relação com estruturas vasculares (Figura 2); em $5(14,3 \%)$ foi útil como guia de procedimentos de ablação tumoral seja por radiofreqüência ou alcoolização, e em $2(5,7 \%)$ foi útil para as duas funções citadas acima.

Em relação à identificação de nódulos, em oito (22,9\%) pacientes a USIO identificou um nódulo a mais em relação aos exames de imagem pré-operatórios ou inspeção e palpação intra-operatória. A Tabela 2 mostra a comparação dos resultados de imagem pré e intra-operatórios, segundo avaliação pelo teste $t$ de Student.

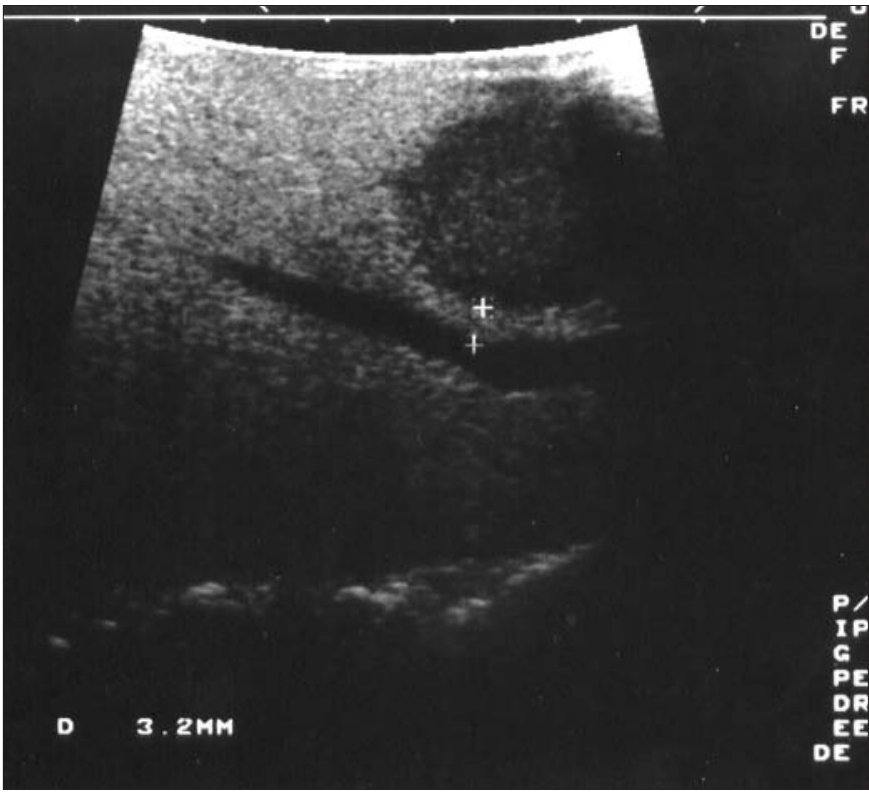

FIGURA 2 - USIO detecta com precisão a pequena distância $(3 \mathrm{~mm})$ entre o nódulo hepático e a veia hepática direita.

TABELA 2 - Análise estatística descritiva segundo o método $t$ de Student na análise do número de nódulos detectado por paciente para cada método de diagnóstico por imagem pré e intra-operatório

\begin{tabular}{|c|c|c|c|c|}
\hline Método & $\mathrm{n}$ & Média (ep) & $P$ & $\operatorname{ricc}(P)$ \\
\hline USIO & 14 & $3,0(0,45)$ & & \\
\hline US & 14 & $1,7(0,29)$ & 0,004 & $0,56(0,036)$ \\
\hline USIO & 30 & $2,73(0,32)$ & & \\
\hline TC & 30 & $1,83(0,19)$ & 0,001 & $0,65(<0,001)$ \\
\hline USIO & 8 & $3,00(0,50)$ & & \\
\hline RNM & 8 & $2,63(0,26)$ & 0,285 & $0,82(0,014)$ \\
\hline USIO & 35 & $2,71(0,28)$ & & \\
\hline $\mathrm{US}+\mathrm{TC}+\mathrm{RNM}$ & 35 & $2,11(0,19)$ & 0,001 & $0,81(<0,001)$ \\
\hline
\end{tabular}

O coeficiente de correlação intra-classe entre cada método diagnóstico pré-operatório e a USIO foi satisfatório, apesar das diferenças significativas entre as médias no número de nódulos encontrados em cada paciente nos exames de US e USIO, e TC e USIO. A RNM e USIO apresentaram média estatisticamente semelhante em relação ao número de nódulos detectados (Figura 3).

Foram considerados como critérios de sensibilidade o exame anatomopatológico ou a evolução clínico-radiológica pósoperatória após 12 semanas da cirurgia e, segundo esses critérios, a USIO deixou de visualizar cinco nódulos. Três deles foram visualizados apenas pelo exame histológico e mediam $0,2 \mathrm{~cm}$, $0,4 \mathrm{~cm}$ e $0,7 \mathrm{~cm}$ e todos tiveram diagnóstico histológico de metástase de adenocarcinoma. $\mathrm{O}$ quarto nódulo não visualizado 


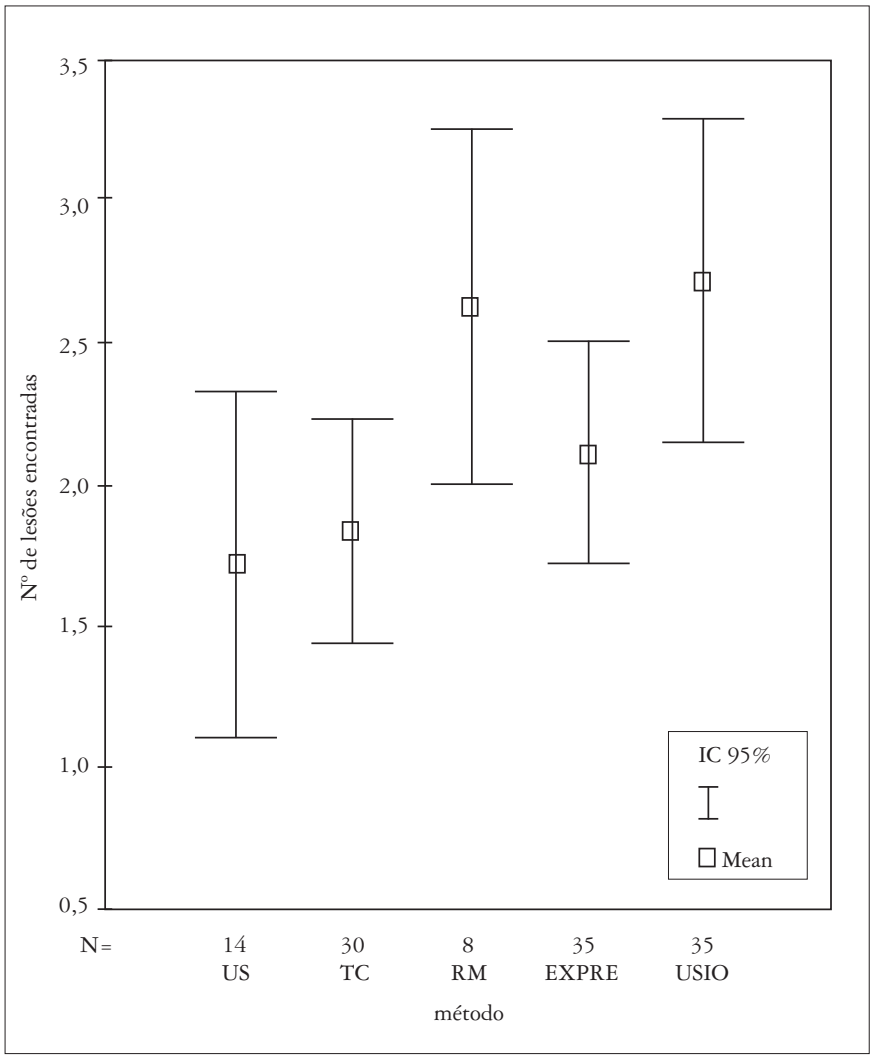

FIGURA 3 - Gráfico da comparação entre as médias de nódulos encontrados pelos métodos de imagem pré e intra-operatório, com intervalo de confiança de $95 \%$

US - ultra-sonografia abdominal; TC - tomografia computadorizada do abdome

RNM - ressonância magnética do abdome; EXPRE - todos os exames pré-operatórios em conjunto; USIO - ultra-sonografia intra-operatória

pela USIO era superficial e foi detectado apenas pela palpação. O quinto nódulo foi detectado 10 semanas após a cirurgia, por controle tomográfico, localizado no segmento VIII e com $1,5 \mathrm{~cm}$ de diâmetro.

Foram detectadas no total 103 lesões focais hepáticas, sendo $78(75,5 \%)$ pelos exames de imagem pré-operatórios, $77(74,8 \%)$ pela inspeção e palpação cirúrgicas, 97 (94,2\%) pela USIO e 3 apenas pelo estudo anatomopatológico.

Cinqüenta e cinco nódulos foram submetidos a análise histológica após a ressecção cirúrgica, 46 não foram ressecados e 2 foram submetidos a ablação tumoral. $\mathrm{O}$ diagnóstico histológico foi de metástase de adenocarcinoma em $41(74,5 \%)$, de melanoma e sarcoma em 4 (7,3\%), tumor neuroendócrino em $3(5,5 \%)$, carcinoma de células claras em $2(3,6 \%)$ e hepatite crônica em $1(1,8 \%)$ paciente.

A sensibilidade da USIO, quando se considerou como parâmetro o estudo anatomopatológico desses 55 nódulos, foi de $94,5 \%$. Em relação às lesões pequenas, avaliadas no presente estudo, levando-se em consideração a heterogeneidade da avaliação pré-operatória para cada paciente, a US foi capaz de detectar $15,0 \%$, a TC $33,3 \%$, a RNM $66,6 \%$ e a USIO $91,6 \%$ das lesões de até $1,5 \mathrm{~cm}$ (Figura 4).

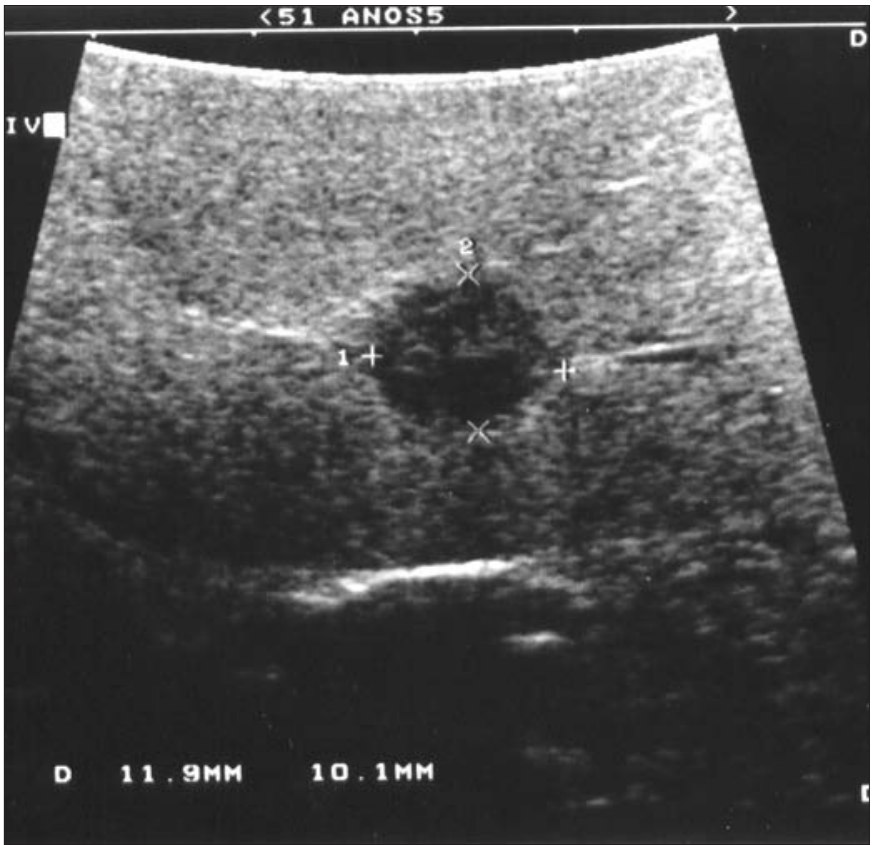

FIGURA 4 - Metástases de adenocarcinoma com 12 mm detectada apenas pela USIO.

\section{DISCUSSÃO}

Nos últimos anos, a ressecção hepática tem sido realizada com freqüência cada vez maior e esta, que no passado era considerada operação de grande risco, apresenta atualmente índices de mortalidade em torno de $3 \%$ em centros especializados ${ }^{(4,6)}$. Deste modo, as indicações de ressecção de metástases hepáticas têm aumentado e encontram-se hoje na literatura, entre outros, relatos de ressecção de metástases de tumores de mama $^{(8,34)}$, carcinoma gástrico $^{(21)}$ e até ovariano ${ }^{(20)}$. Por este motivo realizou-se neste Centro de Tratamento e Pesquisa ressecções de metástases de CCR, tumores neuroendócrinos, renal, melanoma, papila, mama, estromal gástrico e de sarcoma pélvico e, avaliou-se a importância da USIO nessas cirurgias.

Os primeiros estudos citando a importância da USIO datam do final da década de $80^{(19)}$. RIFKIN et al. ${ }^{(25)}$ e BISMUTH et al. ${ }^{(2)}$ relataram que a USIO foi responsável pela mudança do plano cirúrgico em $19 \%$ e $27 \%$ dos pacientes, respectivamente. Apesar da evolução dos métodos de imagem pré-operatórios, vários trabalhos vêm sendo publicados, mostrando que a USIO ainda pode mudar o plano terapêutico em $5 \%$ a $67 \%$ dos $\operatorname{casos}^{(22,}$ 24). Estudos recentes como os de MACHADO ${ }^{(16)}$, no Brasil, ZACHERL et al. ${ }^{(37)}$ e CONLON et al. ${ }^{(5)}$, obtiveram taxas de mudança de plano cirúrgico baseadas em achados da USIO de $22,9 \%, 26,6 \%$ e $18 \%$ dos pacientes, respectivamente ${ }^{(5,16,37)}$.

Na presente casuística a mudança do plano cirúrgico, baseada apenas em informações fornecidas pela USIO, ocorreu em $25,7 \%$ dos pacientes. Além disto, desde os primeiros trabalhos de USIO hepática, os autores enfatizam a acurácia do método em diagnosticar e localizar as lesões focais e suas relações com as estruturas vasculares ${ }^{(2,15,35)}$, pois a USIO permite com maior 
precisão, verificar a distância e/ou o envolvimento de vasos pelas lesões hepáticas, o que é fundamental para orientar a conduta cirúrgica. Neste trabalho, a localização exata das lesões focais hepáticas em relação às estruturas vasculares, através da USIO, ocorreu em cerca de metade $(51,4 \%)$ dos pacientes, pois persistia dúvida baseando-se apenas na avaliação pelos exames de imagem pré-operatórios, especialmente nos tumores localizados nos segmentos IVa e VIII. Afora as alterações no plano cirúrgico, a USIO se prestou para guiar procedimentos de ablação tumoral em quatro pacientes, sendo em dois deles como procedimento isolado e em dois, associada à hepatectomia parcial.

Em 8 dos 35 pacientes (22,8\%) avaliados, a USIO detectou um novo nódulo hepático em relação àqueles já detectados pelos métodos de diagnóstico por imagem pré-operatórios ou pela inspeção e palpação cirúrgicas, e esses mediam entre 0,5 e 1,3 cm. Isto reforça a dificuldade conhecida dos métodos de imagem convencionais em detectar lesões menores que $1,5 \mathrm{~cm}$.

A revisão da literatura, entre 1987 e 2003, mostrou índices de sensibilidade da USIO entre $80 \%$ e $99,3 \%{ }^{(3,18,23,24,36)}$. Os trabalhos que avaliam a sensibilidade da USIO levam em consideração os exames de imagem pré-operatórios, a evolução clínica e radiológica dos pacientes e, em alguns casos, os achados anatomopatológicos. Para o cálculo de sensibilidade é necessário um padrão-ouro e no caso de metástases hepáticas, este é a avaliação histológica. Porém, a patologia como padrão-ouro no estudo do fígado só pode ser usada quando todo o fígado é retirado e analisado, e isso evidentemente não se aplica às hepatectomias por metástases. A análise de sensibilidade fica, dessa forma, reservada às porções ressecadas do fígado.

Nesta série, 55 nódulos em $29(82,8 \%)$ pacientes foram submetidos ao estudo anatomopatológico. Entre eles, 52 (94,5\%) foram detectados pela USIO, demonstrando a alta sensibilidade do método.

Apesar dos avanços tecnológicos na área do diagnóstico por imagem, os índices de sensibilidade da USIO, especialmente quando comparados aos outros exames de imagem, têm se mantido constantes ao longo do tempo e nos diferentes centros onde esta é empregada. É interessante notar que mesmo com esses avanços, a importância da USIO se mantém elevada por mais de 15 anos.

A sensibilidade da USIO na detecção de lesões hepáticas ainda é superior a de outros métodos de diagnóstico por imagem, especialmente para lesões menores que $1,5 \mathrm{~cm}$. MACHI et al. ${ }^{(17)}$, comparando a USIO e as técnicas convencionais para o rastreamento de metástases hepáticas em pacientes com CCR, mostraram que a maioria dos tumores não detectados nos exames pré-operatórios media menos que $2,0 \mathrm{~cm}$. Outros autores compararam a US, a TC, a RNM e o PET scan e mostraram que todas as técnicas têm dificuldade na detecção de lesões menores que $1,0 \mathrm{~cm}$, particularmente as superficiais ${ }^{(11,14,26,28)}$. VALLS et al. ${ }^{(36)}$, avaliando a TC helicoidal na detecção de metástases de CCR, relataram que $21 \%$ das lesões não detectadas pela tomografia tinham entre 0,3 e $1,5 \mathrm{~cm}$.

SCHWARTZ et al. ${ }^{(30)}$ e JANG et al. ${ }^{(12)}$ estudaram a sensibilidade da TC para a detecção de lesões focais hepáticas menores que $1,5 \mathrm{~cm}$ e sua importância na prática clínica, e encontraram que a prevalência de lesões malignas dessas dimensões é pequena. Poder-se-ia, dessa forma, questionar a importância de um método de diagnóstico por imagem que tenha alta sensibilidade para a detecção de lesões focais hepáticas nessas dimensões. Porém, é justamente nos pacientes candidatos a ressecção de metástases hepáticas, que o achado de novas lesões, mesmo pequenas, se faz importante, já que o intuito curativo da cirurgia implica na ressecção completa das lesões malignas, independentemente do número e suas dimensões.

Os exames pré-operatórios nos pacientes desta amostra não foram padronizados, uma vez que muitos deles foram trazidos de outros serviços para avaliação de indicação cirúrgica. A repetição ou realização de novos exames na prática clínica diária do cirurgião, por razões acadêmicas, não é uma solução simples, pois implica em gastos para os pacientes e/ou convênios e o desgaste e demora na condução dos pacientes. Além disso, no caso da TC há os riscos inerentes ao uso de radiação ionizante e contraste iodado.

A falta de padronização dos exames pré-operatórios tanto qualitativa, quanto quantitativa, não permite a avaliação de sensibilidade de cada um desses métodos, porém foi realizada a análise de correlação entre eles e a USIO e encontrou-se um coeficiente de correlação entre cada método de diagnóstico por imagem pré-operatório e a USIO satisfatório (US: $\mathrm{r}=0,56$; TC: $\mathrm{r}=$ 0,65 e RNM: $\mathrm{r}=0,82)$, todos $\operatorname{com} P<0,05$. Esse dado demonstra que, apesar de diferentes, a US, TC e RNM têm resultados satisfatórios na detecção de lesões focais hepáticas. Embora o coeficiente de correlação entre cada método diagnóstico e a USIO ter sido satisfatório, a diferença ente as médias de nódulos identificados pela US e TC, em comparação com a USIO, foi estatisticamente significativa, demonstrando superioridade da USIO sobre esses métodos.

A média de nódulos encontrada na RNM e USIO não mostrou diferença estatística, sugerindo assim, como HAGSPIEGEL et al. ${ }^{(11)}$ e SAHANI et al. ${ }^{(27)}$, que a RNM é o método de diagnóstico por imagem pré-operatório que chega mais perto da avaliação intra-operatória pela USIO porém, neste estudo, foram avaliados apenas oito pacientes com RNM e USIO.

Por outro lado, CONLON et al. ${ }^{(5)}$, comparando os achados intra-operatórios com os da RNM, mostraram mudança de conduta operatória em $18 \%$ dos doentes e novas informações pela USIO em $47 \%$ dos pacientes de sua amostra. Os achados de CONLON et al. ${ }^{(5)}$, empregando RNM pré-operatória, são semelhantes aos de RIFKIN et al. ${ }^{(25)}$ com US, TC e em alguns casos, angiografia e que encontraram novas informações pela USIO em 32,6\% dos casos e mudança do plano cirúrgico em 19\%. Essa comparação mostra que, apesar dos avanços tecnológicos, talvez o impacto dos exames pré-operatórios para o tratamento cirúrgico não tenha mostrado evolução.

KINKEL et al. ${ }^{(13)}$ realizaram uma meta-análise comparando métodos de diagnóstico por imagem não-invasivos na detecção de metástases hepáticas de cânceres do trato gastrointestinal e encontraram sensibilidade de $66 \%$ para a US, $70 \%$ para a TC e $71 \%$ para a RNM. Esses dados confirmam estudos anteriores que, comparando esses diferentes métodos em separado, mostraram semelhança entre eles na detecção das lesões hepáticas.

Há, ainda, grande controvérsia sobre qual o melhor método diagnóstico pré-operatório para detecção de metástases hepáticas. 
VALLS et al. ${ }^{(36)}$ consideram a CT helicoidal o melhor exame, HAGSPIEGEL et al. ${ }^{(11)}$ e SAHANI et al. a RNM ${ }^{(27)}$ e, KINKEL et al. ${ }^{(13)}$ e ROHREN et al. ${ }^{(26)} \mathrm{o}$ PET-scan. O presente estudo não teve como objetivo avaliar qual o método de imagem é o mais indicado na avaliação pré-operatória de pacientes candidatos à ressecção de metástases hepáticas, porém baseando-se nesses achados, sugere-se que a realização de RNM em maior número de pacientes e a análise de seu impacto no estadiamento e na conduta cirúrgica ainda deva ser estudada.

Independentemente do estádio pré-operatório, a USIO é extremamente útil e deve ser utilizada rotineiramente nas cirurgias para ressecção de metástases hepáticas, já que foi fundamental para a condução da cirurgia em $65,7 \%$ dos pacientes desta amostra e mudou o plano cirúrgico original em $25,7 \%$ deles. Estes dados confirmam os encontrados na literatura, de que lesões de até $1,5 \mathrm{~cm}$ são de difícil detecção pela US, TC e RNM e que só a USIO foi capaz de detectar maior número dessas lesões.

Apesar da evolução tecnológica dos métodos de diagnóstico por imagem e índices de sensibilidade altos alcançados por esses métodos, nenhum deles prescinde da USIO durante a ressecção das metástases hepáticas.

Cohen MP, Machado MAC, Herman P. The impact of intra operative ultrasound in metastases liver surgery. Arq Gastroenterol 2005;42(4):206-12.

ABSTRACT - Background - Twenty-five to $50 \%$ of the patients with hepatic metastases are potential candidates for curative surgery. Intraoperative ultrasound has been employed to guide the surgery. Aim - To evaluate this method in liver surgeries and compare it to other imaging methods. Patients and Methods - Thirty-five patients (20 females, with median age of 56 years) with hepatic metastases were prospectively studied between February 2001 and July 2003. Patients had as primary tumors: colorectal cancer (24), neuroendocrine tumors (3), renal cell carcinoma (2), melanoma (2), others (4). Each patient was submitted to at least: computed tomography (30), ultrasonography (14) and magnetic resonance imaging (8). Intraoperative ultrasound was performed in all patients in order to detect liver nodules. The number and location of liver lesions were compared to preoperative results. Results - Intraoperative ultrasound was useful in 23 (65.6\%) of the 35 surgeries and changed the planned surgical strategy in $9(25.7 \%)$. There was a statistical significant correlation between the mean number of nodules identified by ultrasonography, computed tomography, magnetic resonance imaging and intraoperative ultrasound. We found no statistical difference between magnetic resonance imaging and intraoperative ultrasound in identifying hepatic nodules. Fifty-five nodules were submitted to histological evaluation, the gold standard method and 52/55 (94.5\%) were identified by intraoperative ultrasound. Intraoperative ultrasound identified $91,6 \%$ of the smaller than $1.5 \mathrm{~cm}$ lesions, ultrasonography identified $15,0 \%$ of them, computed tomography $33.3 \%$ and magnetic resonance imaging 66,6\%. Conclusions - Intraoperative ultrasound is crucial in the evaluation and decision making in hepatic surgery. Intraoperative ultrasound presents the highest sensibility in the detection of hepatic nodules compared to other imaging methods, especially for small lesions.

HEADINGS - Neoplasm metastasis, ultrasonography. Liver neoplasms, surgery.

\section{REFERÊNCIAS BIBLIOGRÁFICAS}

1. Alves A, Adam R, Majno P, Delvart V, Azoulay D, Castaing D, Bismuth H. Hepatic resection for metastatic renal tumors: is it worthwhile? Ann Surg Oncol 2003;10:705-10

2. Bismuth H, Castaing D, Garden OJ. The use of operative ultrasound in surgery of primary liver tumors. World J Surg 1987; 11:610-4.

3. Cervone A, Sardi A, Conaway GL. Intraoperative ultrasound (IOUS) is essential in the management of metastatic colorectal liver lesions. Am Surg 2000; 66:611-5.

4. Chedid AD, Villwock MM, Chedid MF, Rohde L. Fatores prognósticos na ressecção de metástases hepáticas de câncer colorretal. Arq. Gastroenterol 2003; 40:159-165

5. Conlon R, Jacobs M, Dasgupta D, Lodge JP. The value of intraoperative ultrasound during hepatic resection compared with improved preoperative magnetic resonance imaging. Eur J Ultrasound 2003;16:211-6.

6. Dimick JB, Cowan JA Jr, Knol JA, Upchurch GR Jr. Hepatic resection in the United States: indications, outcomes, and hospital procedural volumes from a nationally representative database. Arch Surg 2003;138:185-91.

7. Elias D, Cavalcanti-de-Albuquerque A, Eggenspieler P, Plaud B, Ducreux M, Spielmann M, Theodore C, Bonvalot S, Lasser P. Resection of liver metastases from a noncolorectal primary: indications and results based on 147 monocentric patients. J Am Coll Surg. 1998; 187:487-93

8. Elias D, Lasser P, Ducreux M, Duvillard P, Ouellet JF, Dromain C, Shlumberger M, Pocard M, Boige V, Miquel C, Baudin E. Liver resection (and associated extrahepatic resections) for metastatic well-differentiated endocrine tumors: a 15-year single center prospective study. Surgery 2003;133:375-82.

9. Fong Y, Blumgart LH, Cohen AM. Surgical treatment of colorectal metastases to the liver. CA Cancer J Clin 1995;45:50-62.
10. Geoghegan JG, Scheele J. Treatment of colorectal liver metastases. Br J Surg 1999;86:158-69.

11. Hagspiel KD, Neidl KF, Eichenberger AC, Weder W, Marincek B. Detection of liver metastases: comparison of superparamagnetic iron oxide-enhanced and unenhanced MR imaging at $1.5 \mathrm{~T}$ with dynamic CT, intraoperative US, and percutaneous US. Radiology 1995; 196:471-8.

12. Jang HJ, Lim HK, Lee WJ, Lee SJ, Yun JY, Choi D. Small hypoattenuating lesions in the liver on single-phase helical CT in preoperative patients with gastric and colorectal cancer: prevalence, significance, and differentiating features. J Comput Assist Tomogr 2002;26:718-24.

13. Kinkel K, Lu Y, Both M, Warren RS, Thoeni RF. Detection of hepatic metastases from cancers of the gastrointestinal tract by using noninvasive imaging methods (US, CT, MR imaging, PET): a meta-analysis. Radiology 2002;224:748-56

14. Knol JA, Marn CS, Francis IR, Rubin JM, Bromberg J, Chang AE. Comparisons of dynamic infusion and delayed computed tomography, intraoperative ultrasound, and palpation in the diagnosis of liver metastases. Am J Surg 1993; 165:81-8.

15. Machado MM, Oliveira IR, Saito OC, Cerri GG. Ultra-sonografia intra-operatória (USIO) do fígado. Radiol Bras 2000;33:15-8.

16. Machado MM. Contribuição da ultra-sonografia intra-operatória (USIO) no estudo do fígado em pacientes candidatos à ressecção hepática por metástases de adenocarcinoma colorretal [tese]. São Paulo: Faculdade de Medicina da Universidade de São Paulo; 2002.

17. Machi J, Isomoto H, Yamashita Y, Kurohiji T, Shirouzu K, Kakegawa T. Intraoperative ultrasonography in screening for liver metastases from colorectal cancer: comparative accuracy with traditional procedures. Surgery 1987;101:678-84.

18. Machi J, Sigel B, Zaren HA, Kurohiji T, Yamashita Y. Operative ultrasonography during hepatobiliary and pancreatic surgery. World J Surg 1993;17:640-6. 
19. Makuuchi M, Torzilli G, Machi J. History of intraoperative ultrasound. Ultrasound Med Biol 1998;24:1229-42.

20. Merideth MA, Cliby WA, Keeney GL, Lesnick TG, Nagorney DM, Podratz KC Hepatic resection for metachronous metastases from ovarian carcinoma. Gynecol Oncol 2003;89:16-21.

21. Okano K, Maeba T, Ishimura K,Karasawa Y. Goda F, Wakabayashi H, Usuki H, Maeta H. Hepatic resection for metastatic tumors from gastric cancer. Ann Surg 2002;235:86-91.

22. Olsen AK. Intraoperative ultrasonography and detection of liver metastases in patients with colorectal cancer. Br J Surg 1990;77:998-9.

23. Paul MA, Mulder LS, Cuesta MA, Sikkenk AC, Lyesen GK, Meijer S. Impact of intraoperative ultrasonography on treatment strategy for colorectal cancer. Br J Surg 1994;81:1660-3.

24. Rafaelsen SR, Kronborg O, Larsen C, Fenger C. Intraoperative ultrasonography in detection of hepatic metastases from colorectal cancer. Dis Colon Rectum 1995;38:355-60.

25. Rifkin MD, Rosato FE, Branch HM. Intraoperative ultrasound of the liver an importan adjunctive tool for decision making in operating room. Ann Surg 1986;205:466-72.

26. Rohren EM, Paulson EK, Hagge R, Wong TZ, Killius J, Clavien PA, Nelson RC. The role of F-18 FDG positron emission tomography in preoperative assessment of liver in patients being considered for curative resection of hepatic metastases from colorectal cancer. Clin Nucl Med 2002;27:550-5.

27. Sahani DV, Kalva SP, Tanabe KK, Hayat SM, O’Neill MJ, Halpern EF, Saini S, Mueller PR. Intraoperative US in patients undergoing surgery for liver neoplasms: comparison with MR imaging. Radiology 2004;232:810-4

28. Said B, McCart JA, Libutti SK, Choyke PL. Ferumoxide-enhanced MRI in patients with colorectal cancer and rising CEA: surgical correlation in early recurrence. Magn Reson Imaging 2000;18:305-9.

29. Sarmiento JM, Heywood G, Rubin J, Ilstrup DM, Nagorney DM, Que FG. Surgical treatment of neuroendocrine metastases to the liver: a plea for resection to increase survival. J Am Coll Surg 2003;197:29-37.

30. Schwartz LH, Gandras EJ, Colangelo SM, Ercolani MC, Panicek DM. Prevalence and importance of small hepatic lesions found at CT in patients with cancer. Radiology 1999;210:71-4.
31. Selzner M, Morse MA, Vredenburgh JJ, Meyers WC, Clavien PA. Liver metastases from breast cancer: long-term survival after curative resection. Surgery 2000;127:383-9.

32. Senettérre E, Taourel P, Bouvier Y, Pradel J, Van Beers B, Daures JP, Pringot J, Mathieu D, Bruel JM. Detection of hepatic metastases: ferumoxides-enhanced MR imaging versus unenhanced MR imaging and CT during arterial portography. Radiology 1996;200:785-92

33. Silas AM, Kruskal JB, Kane RA. Intraoperative ultrasound. Radiol Clin North Am 2001;39:429-48.

34. Spielmann M, Delaloge S. An attempt to clarify indications for hepatectomy for liver metastases from breast cancer. Am J Surg 2003;185:158-64.

35. Torzilli G, Makuuchi M. Tricks for ultrasound-guided resection of colorectal liver metastases. Hepatogastroenterology 2003;50:1-3.

36. Valls C, Andía E, Sánchez A. Hepatic metastases from colorectal cancer: preoperative detection and assessment of resectability with helical CT. Radiology 2002;218:55-60.

37. Zacherl J, Scheuba C, Imhof M, Zacherl M, Langle F, Pokieser P, Wrba F, Wenzl E, Muhbacher F, Steininger R. Current value of intraoperative sonography during surgery for hepatic neoplasms. World J Surg 2002;26:550-4
Recebido em 24/11/2004 Aprovado em 3105/2005 